\title{
Chemical Structure and Sweet Taste of Isocoumarin and Related Compounds. IX ${ }^{1)}$
}

\author{
Masatoshi Yamato, Korchi Sato, Kuniko Hashigaki, \\ and TaKaJi KOYama
}

Faculty of Pharmaceutical Sciences, Okayama University2)

(Received July 5, 1976)

\begin{abstract}
Structural modification of the phenyl moiety (A moiety) of $\beta$-(3-hydroxy-4-methoxyphenyl)ethylbenzene (I), which constitute an essential part of phyllodulcin molecules, was attempted to make the relationship between structure and sweet taste clearer. Twenty-four derivatives of A moiety of I were synthesized, the compound (II, IV, V, VI, X, XXII, XXIII, and XXVI) revealed sweet taste and the other compounds were tasteless. On the basis of these data, the taste of these compounds were discussed in connection with the stereochemical hindrance effect of substituents.
\end{abstract}

Keywords—-phyllodulcin; isocoumarin; sweetness; diphenylethane; structureactivity relationship; effect of bulky substituents

With respect to relationship between the chemical structure and sweet taste of phyllodulcin, we reported that $\beta$-(3-hydroxy-4-methoxyphenyl)ethylbenzene (I) is an essential basic structure for its binding with sweet taste receptor. ${ }^{3)}$ In our subsequent studies, this basic substance I was divided into three moieties (phenyl group was designated as A moiety, ethylene group as B moiety, and 3-hydroxy-4-methoxyphenyl group as C moiety) in order to elucidate how each moiety would contribute to the appearance of sweet taste. A chemical modification of these moieties was made, the relationship between sweet taste and the chemical structure was examined, and some findings on the B moiety were reported. ${ }^{4-6)}$ It has been found that the appearance of sweet taste is controlled by the number and conformation of the methylene group. The present report deals with the effect of replacing various positions with substituents in the $\mathrm{A}$ moiety.

phyllodulcin having an isocoumarin ring, possesses a hydroxyl group corresponding to the A moiety of I and has a strong sweetness. Therefore, A moiety derivatives of I, with a hydroxyl group at the 2,3 , or 4 position, were synthesized. These derivatives were synthesized by the following procedures. Reaction of triphenylphosphine with 2-, 3-, or 4-benzyloxybenzyl chloride gives triphenyl(benzyloxyphenyl)phosphonium chloride, which was condensed with 3-benzyloxy-4-methoxybenzaldehyde by the Wittig reaction, using butyl lithium, and 2- (II), 3- (III), and 4-hydroxy- $\beta$-(3-hydroxy-4-methoxyphenyl)ethylbenzene (IV) were obtained by the catalytic reduction. The compounds II and IV were slightly sweet, but III despite the presence of a hydroxyl group in 3-position showed no sweetness at all. Therefore, in order to prevent ionic dissociation of the hydroxyl group, 2-methoxy- (V), 3-methoxy- (VI), and 4-methoxy- $\beta$-(3-hydroxy-4-methoxyphenyl)ethylbenzene (VII), were

1) Part VIII: M. Yamato, K. Hashigaki, A. Tsukioka, and T. Koyama, Chem. Pharm. Bull. (Tokyo), 25,700 (1977).

2) Location: Tsushima-naka 1-1-1, Okayama, 700, Japan.

3) Part II: M. Yamato, K. Hashigaki, Y. Kuwano, and T. Koyama, Yakugaku Zasshi, 92, 538 (1972).

4) Part III: M. Yamato, T. Kitamura, K. Hashigaki, Y. Kuwano, S. Murakami, and T. Koyama, Yakugaku Zasshi, 92, 850 (1972).

5) Part VI: M. Yamato, K. Hashigaki, J. Uenishi, I. Yamakawa, N. Sato, and T. Koyama, Chem. Pharm. Bull. (Tokyo), 23, 3101 (1975).

6) Part VII: M. Yamato, K. Hashigaki, K. Sato, and T. Koyama, Chem. Pharm. Bull. (Tokyo), 25, 695 (1977). 
synthesized. In this case, $\mathrm{V}$ and VI had a sweet taste but VII was tasteless. These facts have explained the reason why 1,2-bis(3-hydroxy-4-methoxyphenyl)ethane ${ }^{7}$ is not sweet. ${ }^{8}$. Therefore, 4-hydroxy-3-methoxy- $\beta$-(3-hydroxy-4-methoxyphenyl)ethylbenzene (VIII), having a methoxyl group in 3 and hydroxyl group in 4 position of the A moiety in I, each group of which alone taste sweet, was synthesized, but this compound was proved to be tasteless. Moreover, 3,4-dimethoxy- $\beta$-(3-hydroxy-4-methoxyphenyl)ethylbenzene (IX), in which a hydroxyl group in VIII is substituted with a methoxyl group, and 3,4-methylenedioxy- $\beta$ (3-hydroxy-4-methoxyphenyl)ethylbenzene (X), were synthesized. The former was tasteless while the latter was slightly sweet.

Absence of sweetness in 2-carboxy- $\beta$-(3-hydroxy-4-methoxyphenyl)ethylbenzene (XI) was described previously. ${ }^{7)}$ In the present experiment, 3-carboxy- (XII) and 4-carboxy- $\beta$ (3-hydroxy-4-methoxyphenyl)ethylbenzene (XIII), having a carboxyl group in 3 or 4 position of $\mathrm{A}$ moiety in $\mathrm{I}$, were prepared. Both of them proved to be not sweet, indicating that the introduction of a carboxyl group into I eliminates its sweet taste. This fact seems to suggest that the carboxyl group would bind with a basic site other than sweet receptor of the taste bud. Ethoxycarbonyl derivatives, 2- (XIV), 3- (XV), and 4-ethoxycarbonyl- $\beta$ (3-hydroxy-4-methoxyphenyl)ethylbenzene (XVI) were synthesized by esterification of the carboxyl group. The known compound XIV ${ }^{7)}$ had a bitter taste, and XV and XVI were proved to be tasteless. The same facts were further confirmed by the syntheses of 3- (XVII), and 4-methoxycarbonyl- $\beta$-(3-hydroxy-4-methoxyphenyl)ethylbenzene (XVIII).

For the purpose to reduce the lipophilic character of the ester group 2- (XIX), 3- (XX), and 4-hydroxymethyl- $\beta$-(3-hydroxy-4-methoxyphenyl)ethylbenzene (XXI) were synthesized by the reduction of ethoxycarbonyl compounds with lithium aluminum hydride. The compound (XIX), which was reported already, ${ }^{7}$ is slightly sweet while the others were found to be tasteless.

Further reduced type of carboxyl derivatives such as 2- (XXII), 3- (XXIII), and 4-methyl- $\beta$-(3-hydroxy-4-methoxyphenyl)ethylbenzene (XXIV) were synthesized by the Wittig reaction. XXII and XXIII proved to be sweet, but XXIV did not.

From these results the following consideration can be made on the substituent at the position 2 in $\mathrm{A}$ moiety of $\mathrm{I}$. When the intensity of sweetness is not taken into consideration, the compounds having a hydroxyl, methoxyl, or hydroxymethyl group do show sweet taste but not with a carboxyl group, while the ethoxycarbonyl group gives bitter taste.

Phyllodulcin, despite the presence of a lactone ring of an intermolecular ester linkage, has a strong sweet taste. On the other hand, XIV, which is also an ester, has a bitter taste. As to the reason for this, it seems that the difference in the constitution between an intermolecular ester and an intramolecular ester is responsible for it rather than the electrostatic effect of the carbonyl group. In all probability the conformation of the B moiety is greatly effected by the bulky group that has been introduced into the position 2 of the A moiety. For this reason, 2-isopropyl- $\beta$-(3-hydroxy-4-methoxyphenyl)ethylbenzene (XXV) was synthesized, and as was expected, XXV had no sweetness.

An observation has also been made on the effect of a substituent in the 3 and 4 position of the A moiety. Generally, sweet taste was diminished by the introduction of a substituent, especially that of a bulky group in 4 position. 3,4-Methylenedioxy derivative (X) was an exception and it has a faintly sweet taste, but this is assumed to be due whether to the presence of the methylenedioxy group in the same plane as the phenyl group in the A moiety or to the difference of the bulkiness. Therefore, a halogen was introduced into the 4 position. 4-Fluoro- (XXVI), 4-chloro- (XXVII), and 4-bromo- $\beta$-(3-hydroxy-4-

7) Part IV: M. Yamato, K. Sato, K. Hashigaki, T. Ishikawa, and T. Koyama, Yakugaku Zasshi, 93, 1639 (1973).

8) This compound was expected to be sweet but it is actually not sweet. 
methoxyphenyl)ethylbenzene (XXVIII) were synthesized. Both XXVII and XXVIII proved to be tasteless, while XXVI alone tasted faintly sweet.

\section{Discussion}

There are still several unclarified points to be solved about the relationship between sweet and the molecular modification of the A moiety in I, but there is a decreasing tendency of sweetness when the substituents are introduced into the A moiety. The effect of a substituent replaced in 2 position of the A moiety may be summarized as follows. The introduction of small groups such as hydroxyl, methyl, methoxyl, and hydroxymethyl groups. into 2 position of the A moiety gives a sweet taste. However, the introduction of a bulky isopropyl group resulted in the loss of sweetness and similarly, bulky ethoxycarbonyl group rather induces a bitter taste. This fact may be interpreted to result from the repulsion between the bulky group and the B moiety, and consequently, making it difficult to bind. these derivatives with the sweet taste receptor.

Hydroxyl group in 3 position of A moiety in I corresponds to the hydroxyl group at 8 : position of phyllodulcin. Nevertheless, the 3 position of I had no sweetness, since it is not a special site for appearance of a sweet taste. The derivatives of I having a hydroxyl group in 2 or 4 position rather than the 3 position reveal a sweet taste. These facts appear to be contradictory but, as will be clear from IR spectrum of phyllodulcin, hydroxyl group formed a strong hydrogen bond with carbonyl group of lactone ring $\left(v_{\mathrm{C}=0} 1685 \mathrm{~cm}^{-1}\right),{ }^{9)}$ which seems to have weakened the nature of the hydroxyl group.

when a carboxyl or an ester group is introduced into the A moiety, the substance becomes tasteless or bitter. Essentially, the A moiety is the site where the taste bud receptor is bound by the Van der Waal's force. However, these groups introduced there protrude from the same plane of the phenyl group and as a result, the A moiety and the receptor are bound only in a deformed shape. For this reason, it seems that the bound position of $\mathrm{B}$ and $\mathrm{C}$ moiety slips away from the surface of the receptor. The same thing can be seen in the non-sweet IX and sweet X. Namely, when the introduced substituents are appropriately. small and they do not slip out of the same plane of the phenyl group, these derivatives of I seem to reveal a sweet taste.

As for the substituent at 4 position, all the bulky groups eliminate sweet taste. Especially, an interesting result was obtained in the appearance of sweetness with a halogen group. Fluorine has the smallest atomic radius in the halogen group, while chlorine and bromine have a larger atomic radius than fluorine. Consequently, fluorine derivative XXVI tastes sweet but XXVII (chlorine derivative) and XXVIII (bromine derivative) not sweet.

\section{Experimentai ${ }^{10)}$}

Synthesis of Phosphonium Salts-An equimolecular mixture of a benzyl chloride and triphenylphosphine in dry xylene (or dry benzene) was heated on a water bath. After cooling, the precipitate was recrystallized from acetone or ethanol. The results were shown in Table I.

Synthesis of 3'-Benzyloxy-4'-methoxystilbenes-Method A: A suspension of a powdered phosphonium. salt in dry ether was kept below $5^{\circ}$ under nitrogen, butyl lithium ( $15 \%$ solution in hexane) was added with stirring. After $10 \mathrm{~min}, 3$-benzyloxy-4-methoxybenzaldehyde was added and stirred for $1 \mathrm{hr}$. Then, the ice bath was removed and stirring was continued for $4 \mathrm{hr}$ at room temperature. The precipitate was separated and treated with hot methanol, leaving crystalline product. The same substance was obtained from the ether layer and recrystallized from ethanol or ethanol-chloroform mixture. The results were shown in Table II.

Method B: To an absolute methanol solution of equimolecular phosphonium salt and 3-benzyloxy-4methoxybenzaldehyde, a methanolic solution of $0.2 \mathrm{~N}$ lithium methoxide was added dropwise under nitrogen. After stirring $5 \mathrm{hr}$ the precipitate was crystallized from ethanol-chloroform mixture. The results were shown. in Table III.

9) M. Yamato, Yakugaku Zasshi, 79, 1069 (1959).

10) All melting points were measured on a micro hot-stage apparatus and are uncorrected. 
TABLE I. Synthesis of Phosphonium Salts

\begin{tabular}{|c|c|c|c|c|c|c|}
\hline \multicolumn{3}{|c|}{ Materials } & \multirow[b]{2}{*}{$\begin{array}{l}\text { Solvent } \\
\text { (ml) }\end{array}$} & \multirow[b]{2}{*}{$\underset{(\mathrm{hr})}{\left.\mathrm{R} \cdot{ }^{a}\right)}$} & \multirow[b]{2}{*}{$\operatorname{mp}_{\left({ }^{\circ} \mathrm{C}\right)}$} & \multirow[b]{2}{*}{$\begin{array}{l}\text { Yield } \\
(\%)\end{array}$} \\
\hline R- & $\mathrm{x}$ & $\begin{array}{l}\mathrm{Ph}_{3} \mathrm{P} \\
(\mathrm{g})\end{array}$ & & & & \\
\hline $\begin{array}{l}2-\mathrm{OCH}_{2} \mathrm{Ph} \\
17.5\end{array}$ & $\mathrm{Cl}$ & 19.6 & $\begin{array}{l}\text { benzene } \\
30\end{array}$ & 15 & $225-226$ & 86.5 \\
\hline $\begin{array}{l}3-\mathrm{OCH}_{2} \mathrm{Ph} \\
12.6\end{array}$ & $\mathrm{Cl}$ & 14.3 & $\begin{array}{l}\text { benzene } \\
30\end{array}$ & 24 & $249-250$ & 74.6 \\
\hline $\begin{array}{c}4-\mathrm{OCH}_{2} \mathrm{Ph} \\
5.9\end{array}$ & $\mathrm{Cl}$ & 6.7 & $\begin{array}{c}\text { benzene } \\
15\end{array}$ & 14 & $248-251$ & 68.6 \\
\hline $\begin{array}{c}2-\mathrm{OCH}_{3} \\
4.0\end{array}$ & $\mathrm{Cl}$ & 5.9 & $\begin{array}{c}\text { benzene } \\
15\end{array}$ & 15 & $249-251$ & 92.5 \\
\hline $\begin{array}{c}3-\mathrm{OCH}_{3} \\
7.8\end{array}$ & $\mathrm{Cl}$ & 13.1 & $\begin{array}{c}\text { benzene } \\
20\end{array}$ & 24 & $278-279$ & 66.6 \\
\hline $\begin{array}{c}4-\mathrm{OCH}_{3} \\
11.9\end{array}$ & $\mathrm{Cl}$ & 19.8 & $\begin{array}{c}\text { benzene } \\
40\end{array}$ & 2 & $245-247$ & 51.0 \\
\hline $\begin{array}{c}3-\mathrm{OCH}_{3} \\
4-\mathrm{OCH}_{2} \mathrm{Ph} \\
7.0\end{array}$ & $\mathrm{Cl}$ & 7.0 & $\begin{array}{l}\text { benzene } \\
20\end{array}$ & 24 & $245-248$ & 57.2 \\
\hline $\begin{array}{l}3,4-\mathrm{di}-\mathrm{OCH}_{3} \\
3.0\end{array}$ & $\mathrm{Cl}$ & 4.2 & $\begin{array}{c}\text { xylene } \\
20\end{array}$ & 7 & $237-239$ & 74.0 \\
\hline $\begin{array}{l}3-\mathrm{O}>\mathrm{CH}_{2} \\
3-\mathrm{O}>10.0\end{array}$ & $\mathrm{Cl}$ & 15.4 & $\begin{array}{c}\text { xylene } \\
20\end{array}$ & 20 & $241-242$ & 74.9 \\
\hline $\begin{array}{c}2-\mathrm{CH}_{3} \\
10.0\end{array}$ & $\mathrm{Cl}$ & 19.0 & $\begin{array}{l}\text { xylene } \\
20\end{array}$ & 12 & $279-281$ & 82.5 \\
\hline $\begin{array}{c}3-\mathrm{CH}_{3} \\
10.0\end{array}$ & $\mathrm{Cl}$ & 19.0 & $\begin{array}{l}\text { benzene } \\
20\end{array}$ & 20 & 300 & 76.8 \\
\hline $\begin{array}{r}4-\mathrm{CH}_{3} \\
3.6\end{array}$ & $\mathrm{Cl}$ & 6.7 & $\begin{array}{c}\text { xylene } \\
20\end{array}$ & 2 & $262-265$ & 70.0 \\
\hline $\begin{array}{c}3-\mathrm{COOCH}_{3} \\
19.4\end{array}$ & $\mathrm{Br}$ & 22.2 & $\begin{array}{c}\text { xylene } \\
50\end{array}$ & 10 & $231-233$ & 68.5 \\
\hline $\begin{array}{l}4-\mathrm{COOCH}_{3} \\
11.5\end{array}$ & $\mathrm{Br}$ & 13.1 & $\begin{array}{l}\text { xylene } \\
20\end{array}$ & 10 & $236-238$ & 64.3 \\
\hline $4-F_{4.5}$ & $\mathrm{Br}$ & 6.2 & $\begin{array}{l}\text { benzene } \\
20\end{array}$ & 3 & 300 & 97.8 \\
\hline $\begin{array}{l}4-\mathrm{Cl} \\
8.0\end{array}$ & $\mathrm{Br}$ & 10.0 & non solvent & 1 & $284-287$ & 88.9 \\
\hline $\begin{array}{r}4-\mathrm{Br} \\
6.8\end{array}$ & $\mathrm{Cl}$ & 8.8 & $\begin{array}{l}\text { xylene } \\
20\end{array}$ & 24 & $268-269$ & 45.5 \\
\hline
\end{tabular}

a) reaction time

Synthesis of $\boldsymbol{\beta}$-(3-Hydroxy-4-methoxyphenyl)ethylbenzenes-A suspension of a $3^{\prime}$-benzyloxy-4'methoxystilbene in acetic acid was catalytically reduced with $10 \% \mathrm{Pd} / \mathrm{C}$. After calculated amount of hydrogen was absorbed, the solvent was distilled off in vacuo. The residue was dissolved in ethyl acetate and washed with saturated potassium bicarbonate and water. The solvent was removed and the residue was purified on a column (silica gel) with dichloromethane. The results were shown in Table IV.

3-Carboxy- $\beta$-(3-hydroxy-4-methoxyphenyl)ethylbenzene (XII)-A suspension of XVII $(0.3 \mathrm{~g})$, prepared as above, in the mixture of $10 \mathrm{ml}$ of $10 \%$ potassium hydroxide aqueous solution and $10 \mathrm{ml}$ of ethanol was heated at $80^{\circ}$ until the crystal was dissolved. After cooling, the mixture was acidified with $10 \%$ sulfuric acid. The solid product was obtained and recrystallized from ethanol. Colorless needles, mp $169-172^{\circ}$, $0.15 \mathrm{~g}(51.5 \%)$.

4-Carboxy- $\boldsymbol{\beta}$-(3-hydroxy-4-methoxyphenyl)ethylbenzene (XIII)—-It was obtained from XVIII (2g) by the same method as in XII. The product was recrystallized from a large quantity of methanol. Colorless needles, $\mathrm{mp} 234-235^{\circ}, 1.5 \mathrm{~g}(79.0 \%)$.

3-Ethoxycarbonyl- $\beta$-(3-hydroxy-4-methoxyphenyl)ethylbenzene (XV)—To a suspension of XII (0.5 g) in $10 \mathrm{ml}$ of absolute ethanol, 3 drops of conc. sulfuric acid were added and heated for $4 \mathrm{hr}$. After heating, the solvent was evaporated, and the residue was dissolved in ethyl acetate, and washed with saturated 
TABle II. Synthesis of $3^{\prime}$-Benzyloxy-4'-methoxystilbenes by Method A

\begin{tabular}{|c|c|c|c|c|c|c|}
\hline & \multicolumn{2}{|c|}{ Phosphonium salt } & \multirow{2}{*}{$\begin{array}{l}\text { But-Li }{ }^{a)} \\
\quad(\mathrm{ml})\end{array}$} & \multirow{2}{*}{$\begin{array}{c}\text { Benzyliso- } \\
\text { vanillin } \\
(g)\end{array}$} & \multirow{2}{*}{$\operatorname{mp}_{\left({ }^{\circ} \mathrm{C}\right)}$} & \multirow{2}{*}{$\begin{array}{c}\text { Yield } \\
(\%)\end{array}$} \\
\hline & R- & (g) & & & & \\
\hline & $2-\mathrm{OCH}_{2} \mathrm{Ph}$ & 7.4 & 7.0 & 3.6 & $127-128$ & 40.7 \\
\hline & $3-\mathrm{OCH}_{2} \mathrm{Ph}$ & 23.0 & 29.5 & 11.1 & $144-145$ & 36.0 \\
\hline & $4-\mathrm{OCH}_{2} \mathrm{Ph}$ & 19.8 & 25.4 & 9.4 & $143-144$ & 29.3 \\
\hline & $2-\mathrm{OCH}_{3}$ & 9.9 & 15.4 & 5.7 & $144-145$ & 44.2 \\
\hline & $3-\mathrm{OCH}_{3}$ & 8.3 & 12.7 & 4.8 & $140-145$ & 15.0 \\
\hline & $4-\mathrm{OCH}_{3}, \ldots$ & $\therefore 8.3$ & 12.7 & 4.8 & $131-132$ & 26.2 \\
\hline & $\begin{array}{l}3-\mathrm{OCH}_{3} \\
4-\mathrm{OCH}_{2} \mathrm{Ph}^{2}\end{array}$ & 8.0 & 9.2 & 4.0 & $134-137$ & 3.4 \\
\hline & 3,4-Methylenedioxy & 8.7 & 12.4 & 4.8 & $120-123$ & 41.7 \\
\hline & $3,4-\mathrm{di}^{-} \mathrm{OCH}_{3}$ & 7.4 & 10.8 & 4.4 & $171-173$ & 52.0 \\
\hline & $2-\mathrm{CH}_{3}$ & 12.1 & 19.1 & 7.3 & $106-108$ & 58.6 \\
\hline & $3-\mathrm{CH}_{3}$ & 8.1 & 12.5 & 4.8 & $121-123$ & 66.6 \\
\hline & $4-\mathrm{CH}_{3}$ & 8.1 & 12.5 & 4.8 & $138-141$ & 55.0 \\
\hline
\end{tabular}

a) butyl lithium ( $15 \%$ solution in hexane)

TABLE III. Synthesis of 3'-Benzyloxy-4'-methoxystilbenes by Method B

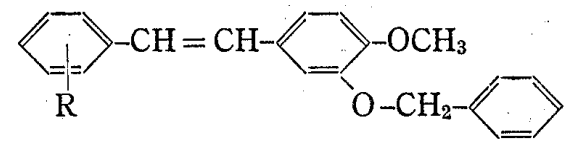

\begin{tabular}{lrcrrr}
\hline \multicolumn{2}{r}{ Phosphonium salt } & $\begin{array}{c}\text { Benzyliso- } \\
\text { vanillin } \\
(\mathrm{g})\end{array}$ & $\begin{array}{c}\mathrm{Li} \\
(\mathrm{g})\end{array}$ & $\begin{array}{c}\text { mp } \\
\left({ }^{\circ} \mathrm{C}\right)\end{array}$ & $\begin{array}{c}\text { Yield } \\
(\%)\end{array}$ \\
\hline $3-\mathrm{COOCH}_{3}$ & 9.8 & 4.9 & 0.174 & $94-96$ & 16.1 \\
$4-\mathrm{COOCH}_{3}$ & 9.8 & 4.9 & 0.174 & $120-125$ & 26.9 \\
$4-\mathrm{F} a)$ & 10.0 & 5.4 & 0.157 & $118-119$ & 5.4 \\
$4-\mathrm{Cl}$ & 15.0 & 7.8 & 0.224 & $141-143$ & 31.1 \\
$4-\mathrm{Br}^{b}$ & 7.0 & 3.6 & 0.105 & $155-156$ & 39.0 \\
\hline
\end{tabular}

a) The product was very soluble in methanol, hence it was purified by extracting with pet. ether and on a column (silica gel) with dichloromethane.

b) Phosphonium chloride was used.

potassium bicarbonate and water. Ethyl acetate was evaporated and the residue was purified on a column (silica gel) with dichloromethane. It was recrystallized from $50 \%$ ethanol. Colorless prisms, $\mathrm{mp} 36-36.5^{\circ}$, $0.3 \mathrm{~g}(51.0 \%)$. When ethanol was used as solvent in the reaction of triphenyl-3-methoxycarbonylbenzylphosphonium bromide in method $\mathrm{B}$, an ester interchange took place to yield 3-ethoxycarbonyl-3'-benzyloxy$4^{\prime}$-methoxystilbene $\left(\operatorname{mp} 91-93^{\circ}, 54.2 \%\right)$. XV was obtained also by reducing this compound $(54.9 \%)$.

4-Ethoxycarbonyl- $\beta$-(3-hydroxy-4-methoxyphenyl)ethylbenzene (XVI)_-It was obtained from XIII $(0.5 \mathrm{~g})$ by the same method as in XV. The product was recrystallized from $80 \%$ ethanol. Colorless needles, $\mathrm{mp} 72-73^{\circ}, 0.5 \mathrm{~g}(61.2 \%)$.

3-Hydroxymethyl- $\beta$-(3-hydroxy-4-methoxyphenyl)ethylbenzene (XX)—A suspension of XVII (0.3 g) in a mixture of $20 \mathrm{ml}$ of benzene and $10 \mathrm{ml}$ of dry dioxane was added dropwise to the suspension of lithium aluminum hydride $(0.1 \mathrm{~g})$ in $20 \mathrm{ml}$ of dry ether. After stirring at room temperature for $4 \mathrm{hr}$, the reaction mixture was hydrolyzed in a usual way. The ether layer was separated and washed with $10 \%$ potassium carbonate and water. The ether was evaporated, and the residue was purified by column chromatography using silica gel and dichloromethane-ether. It was recrystallized from $80 \%$ ethanol. Colorless needles, mp $84-85^{\circ}, 0.1 \mathrm{~g}(37.0 \%)$.

4-Hydroxymethyl- $\beta$-(3-hydroxy-4-methoxyphenyl)ethylbenzene (XXI)_- It was obtained from XVIII $(1 \mathrm{~g})$ by the same method as in XX. The product was recrystallized from $70 \%$ ethanol. Colorless needles, $\operatorname{mp} 151-152^{\circ}, 0.5 \mathrm{~g}(59.9 \%)$. 
TABLE IV. Synthesis of $\beta$-(3-Hydroxy-4-methoxyphenyl)ethylbenzenes<smiles>[R]c1cccc(CCc2ccc(OC)c(O)c2)c1</smiles>

\begin{tabular}{|c|c|c|c|c|c|}
\hline \multicolumn{2}{|c|}{ Product } & \multirow{2}{*}{$\begin{array}{l}\text { Stilbene } \\
(\mathrm{g})\end{array}$} & \multirow{2}{*}{$\begin{array}{c}\mathrm{CH}_{3} \mathrm{COOH} \\
(\mathrm{ml})\end{array}$} & \multirow{2}{*}{$\begin{array}{c}\operatorname{mp}\left({ }^{\circ} \mathrm{C}\right) \\
\text { or } \mathrm{bp}\end{array}$} & \multirow{2}{*}{$\begin{array}{l}\text { Yield } \\
(\%)\end{array}$} \\
\hline No. & $\mathrm{R}-$ & & & & \\
\hline II & $2-\mathrm{OH}$ & 2.4 & 100 & $91-92$ & 57.4 \\
\hline III & $3-\mathrm{OH}$ & 2.3 & 100 & $157-158$ & $81: 2$ \\
\hline IV & $4-\mathrm{OH}$ & 4.8 & 200 & $116-117$ & 79.1 \\
\hline $\mathrm{V}$ & $2-\mathrm{OCH}_{3}$ & 3.6 & 200 & $180 / 3 \mathrm{mmHg}$ & 22.4 \\
\hline VI & $3-\mathrm{OCH}_{3}$ & 1.03 & 50 & $82-83$ & 13.0 \\
\hline VII & $4-\mathrm{OCH}_{3}$ & 1.8 & 100 & $116-117$ & 33.6 \\
\hline VIII & $\begin{array}{l}3-\mathrm{OCH}_{3} \\
4-\mathrm{OH}\end{array}$ & 0.25 & 20 & $180 / 4 \mathrm{mmHg}$ & 33.3 \\
\hline IX & $3,4-\mathrm{di}-\mathrm{OCH}_{3}$ & 1.3 & 50 & $103-104$ & 11.1 \\
\hline $\mathrm{X}$ & $\begin{array}{l}3-\mathrm{O} \\
4-\mathrm{O}\end{array} \mathrm{CH}_{2}$ & 3.0 & 200 & $108-109$ & 24.3 \\
\hline XVII & $3-\mathrm{COOCH}_{3}$ & 1.2 & 100 & $82-83$ & 35.0 \\
\hline XVIII & $4-\mathrm{COOCH}_{3}$ & 2.0 & 100 & $112-113$ & 58.0 \\
\hline XXII & $2-\mathrm{CH}_{3}$ & 5.8 & 200 & $69-70$ & 64.2 \\
\hline XXIII & $3-\mathrm{CH}_{3}$ & 4.0 & 100 & $88-89$ & 80.0 \\
\hline XXIV & $4-\mathrm{CH}_{3}$ & 3.0 & 100 & $122-123$ & 70.0 \\
\hline XXVI & $4-F$ & 0.4 & 80 & $63-64$ & 34.5 \\
\hline XXVII & $4-\mathrm{Cl}$ & 3.5 & 200 & $71-72$ & 80.2 \\
\hline XXVIII & $4-\mathrm{Br}$ & 2.3 & 150 & $86-87$ & 61.5 \\
\hline
\end{tabular}

TABLE V. Elemental Analysis of $\beta$-(3-Hydroxy-4-methoxyphenyl)ethylbenzenes

\begin{tabular}{|c|c|c|c|c|c|c|}
\hline \multirow{3}{*}{ No. } & \multirow{3}{*}{ R- } & \multirow{3}{*}{ Formula } & \multicolumn{4}{|c|}{ Analysis (\%) } \\
\hline & & & \multicolumn{2}{|c|}{ Calcd. } & \multicolumn{2}{|c|}{ Found } \\
\hline & & & $\mathrm{C}$ & $\mathrm{H}$ & $\mathrm{C}$ & $\mathrm{H}$ \\
\hline II $a$ ) & $2-\mathrm{OH}$ & $\mathrm{C}_{15} \mathrm{H}_{16} \mathrm{O}_{3}$ & 73.75 & 6.60 & 73.52 & 6.55 \\
\hline III & $3-\mathrm{OH}$ & $\mathrm{C}_{15} \mathrm{H}_{16} \mathrm{O}_{3}$ & 73.75 & 6.60 & 73.48 & 6.70 \\
\hline $\operatorname{IV} a)$ & $4-\mathrm{OH}$ & $\mathrm{C}_{15} \mathrm{H}_{16} \mathrm{O}_{3}$ & 73.75 & 6.60 & 73.78 & 6.74 \\
\hline$V^{a} a$ & $2-\mathrm{OCH}_{3}$ & $\mathrm{C}_{16} \mathrm{H}_{18} \mathrm{O}_{3}$ & 74.39 & 7.02 & 74.15 & 6.88 \\
\hline $\mathrm{VI}(a)$ & $3-\mathrm{OCH}_{3}$ & $\mathrm{C}_{16} \mathrm{H}_{18} \mathrm{O}_{3}$ & 74.39 & 7.02 & 74.40 & 6.92 \\
\hline VII & $4-\mathrm{OCH}_{3}$ & $\mathrm{C}_{16} \mathrm{H}_{18} \mathrm{O}_{3}$ & 74.39 & 7.02 & 74.16 & 6.99 \\
\hline VIII & $\begin{array}{l}3-\mathrm{OCH}_{3} \\
4-\mathrm{OH}\end{array}$ & $\mathrm{C}_{16} \mathrm{H}_{18} \mathrm{O}_{4}$ & 70.05 & 6.61 & 70.12 & 6.69 \\
\hline IX & $3,4-\mathrm{di}-\mathrm{OCH}_{3}$ & $\mathrm{C}_{17} \mathrm{H}_{20} \mathrm{O}_{4}$ & 70.81 & 6.99 & 71.11 & 7.08 \\
\hline $\mathrm{X}^{a)}$ & $\begin{array}{l}3-\mathrm{O} \\
4-\mathrm{O}\end{array}>\mathrm{CH}_{2}$ & $\mathrm{C}_{16} \mathrm{H}_{16} \mathrm{O}_{4}$ & 70.57 & 5.92 & 70.42 & 5.94 \\
\hline XII & $3-\mathrm{COOH}$ & $\mathrm{C}_{16} \mathrm{H}_{16} \mathrm{O}_{4}$ & 70.57 & 5.92 & 70.22 & 5.83 \\
\hline XIII & $4-\mathrm{COOH}$ & $\mathrm{C}_{16} \mathrm{H}_{16} \mathrm{O}_{4}$ & 70.57 & 5.92 & 70.34 & 5.91 \\
\hline $\mathrm{XV}$ & $3-\mathrm{COOC}_{2} \mathrm{H}_{5}$ & $\mathrm{C}_{18} \mathrm{H}_{20} \mathrm{O}_{4}$ & 71.98 & 6.71 & 71.89 & 6.66 \\
\hline$X V I$ & $4-\mathrm{COOC}_{2} \mathrm{H}_{5}$ & $\mathrm{C}_{18} \mathrm{H}_{20} \mathrm{O}_{4}$ & 71.98 & 6.71 & 71.98 & 6.77 \\
\hline XVII & $3-\mathrm{COOCH}_{3}$ & $\mathrm{C}_{17} \mathrm{H}_{18} \mathrm{O}_{4}$ & 71.31 & 6.34 & 71.24 & 6.09 \\
\hline XVIII & $4-\mathrm{COOCH}_{3}$ & $\mathrm{C}_{17} \mathrm{H}_{18} \mathrm{O}_{4}$ & 71.31 & 6.34 & 71.03 & 6.31 \\
\hline $\mathrm{XX}$ & $3-\mathrm{CH}_{2} \mathrm{OH}$ & $\mathrm{C}_{16} \mathrm{H}_{18} \mathrm{O}_{3}$ & 74.39 & 7.02 & 74.28 & 7.07 \\
\hline XXI & $4-\mathrm{CH}_{2} \mathrm{OH}$ & $\mathrm{C}_{16} \mathrm{H}_{18} \mathrm{O}_{3}$ & 74.39 & 7.02 & 74.28 & 7.12 \\
\hline $\mathrm{XXI} a$ ) & $2-\mathrm{CH}_{3}$ & $\mathrm{C}_{16} \mathrm{H}_{18} \mathrm{O}_{2}$ & 79.31 & 7.49 & 79.57 & 7.37 \\
\hline XXIII $a$ ) & $3-\mathrm{CH}_{3}$ & $\mathrm{C}_{16} \mathrm{H}_{18} \mathrm{O}_{2}$ & 79.31 & 7.49 & 79.56 & 7.49 \\
\hline XXIV & $4-\mathrm{CH}_{3}$ & $\mathrm{C}_{16} \mathrm{H}_{18} \mathrm{O}_{2}$ & 79.31 & 7.49 & 79.14 & 7.44 \\
\hline XXV & $2-\mathrm{CH}\left\langle\mathrm{CH}_{3}^{\mathrm{CH}_{3}}\right.$ & $\mathrm{C}_{18} \mathrm{H}_{22} \mathrm{O}_{2}$ & 79.96 & 8.20 & 79.60 & 8.10 \\
\hline $\mathrm{XXVI} a)$ & $4-F$ & $\mathrm{C}_{15} \mathrm{H}_{15} \mathrm{O}_{2} \mathrm{~F}$ & 73.17 & 6.10 & 73.25 & 6.07 \\
\hline XXVII & $4-\mathrm{Cl}$ & $\mathrm{C}_{15} \mathrm{H}_{15} \mathrm{O}_{2} \mathrm{Cl}$ & 68.57 & 5.71 & 68.48 & 6.09 \\
\hline XXVIII & $4-\mathrm{Br}$ & $\mathrm{C}_{15} \mathrm{H}_{15} \mathrm{O}_{2} \mathrm{Br}$ & 58.63 & 4.89 & 58.67 & 4.92 \\
\hline
\end{tabular}


TABLE VI. NMR Spectra of $\beta$-(3-Hydroxy-4-methoxyphenyl)ethylbenzenes ${ }^{a)}$

\begin{tabular}{|c|c|}
\hline No. & $\mathrm{NMR}(\delta)$ in $\mathrm{CDCl}_{3}$ \\
\hline II & $\begin{array}{l}2.84\left(4 \mathrm{H}, \mathrm{s}, \mathrm{CH}_{2}-\mathrm{CH}_{2}\right), 3.85\left(3 \mathrm{H}, \mathrm{s}, \mathrm{OCH}_{3}\right), 4.70(1 \mathrm{H}, \text { broad, } \mathrm{OH}), 5.63(1 \mathrm{H}, \text { broad, } \mathrm{OH}), 7.25- \\
6.60(7 \mathrm{H}, \mathrm{m} \text {, aromatic } \mathrm{H}) .\end{array}$ \\
\hline III & $\begin{array}{l}2.80\left(4 \mathrm{H}, \mathrm{s}, \mathrm{CH}_{2}-\mathrm{CH}_{2}\right), 3.85\left(3 \mathrm{H}, \mathrm{s}, \mathrm{OCH}_{3}\right), 4.70(1 \mathrm{H}, \text { broad, } \mathrm{OH}), 5.55(1 \mathrm{H}, \mathrm{s}, \mathrm{OH}), 7.24-6.58 \\
(7 \mathrm{H}, \mathrm{m} \text {, aromatic } \mathrm{H}) .\end{array}$ \\
\hline IV & $\begin{array}{l}2.79\left(4 \mathrm{H}, \mathrm{s}, \mathrm{CH}_{2}-\mathrm{CH}_{2}\right), 3.86\left(3 \mathrm{H}, \mathrm{s}, \mathrm{OCH}_{3}\right), 5.75-4.40(2 \mathrm{H}, \text { broad, } \mathrm{OH} \times 2), 7.10-6.57(7 \mathrm{H}, \mathrm{m} \text {, } \\
\text { aromatic } \mathrm{H}) \text {. }\end{array}$ \\
\hline V & $\begin{array}{l}2.78\left(4 \mathrm{H}, \mathrm{s}, \mathrm{CH}_{2}-\mathrm{CH}_{2}\right), 3.78\left(3 \mathrm{H}, \mathrm{s}, \mathrm{OCH}_{3}\right), 3.81\left(3 \mathrm{H}, \mathrm{s}, \mathrm{OCH}_{3}\right), 5.50(1 \mathrm{H}, \mathrm{s}, \mathrm{OH}), 7.25-6.57 \\
(7 \mathrm{H}, \mathrm{m} \text {, aromatic } \mathrm{H}) .\end{array}$ \\
\hline VI & $\begin{array}{l}2.79\left(4 \mathrm{H}, \mathrm{s}, \mathrm{CH}_{2}-\mathrm{CH}_{2}\right), 3.73\left(3 \mathrm{H}, \mathrm{s}, \mathrm{OCH}_{3}\right), 3.80\left(3 \mathrm{H}, \mathrm{s}, \mathrm{OCH}_{3}\right), 5.54(1 \mathrm{H}, \mathrm{s}, \mathrm{OH}) 7.26-6.50 \\
(7 \mathrm{H}, \mathrm{m} \text {, aromatic } \mathrm{H}) .\end{array}$ \\
\hline VII & $\begin{array}{l}2.79\left(4 \mathrm{H}, \mathrm{s}, \mathrm{CH}_{2}-\mathrm{CH}_{2}\right), 3.77\left(3 \mathrm{H}, \mathrm{s}, \mathrm{OCH}_{3}\right), 3.85\left(3 \mathrm{H}, \mathrm{s}, \mathrm{OCH}_{3}\right), 5.56(1 \mathrm{H}, \mathrm{s}, \mathrm{OH}), 7.25-6.55 \\
(7 \mathrm{H}, \mathrm{m} \text {, aromatic } \mathrm{H}) .\end{array}$ \\
\hline VIII & $\begin{array}{l}2.72\left(4 \mathrm{H}, \mathrm{s}, \mathrm{CH}_{2}-\mathrm{CH}_{2}\right), 3.75\left(3 \mathrm{H}, \mathrm{s}, \mathrm{OCH}_{3}\right), 3.78\left(3 \mathrm{H}, \mathrm{s}, \mathrm{OCH}_{3}\right), 5.39(1 \mathrm{H}, \text { broad, } \mathrm{OH}), 5.49 \\
(1 \mathrm{H}, \text { broad, } \mathrm{OH}), 6.80-6.49(7 \mathrm{H}, \mathrm{m} \text {, aromatic } \mathrm{H}) .\end{array}$ \\
\hline IX & $\begin{array}{l}2.80\left(4 \mathrm{H}, \mathrm{s}, \mathrm{CH}_{2}-\mathrm{CH}_{2}\right), 3.80\left(3 \mathrm{H}, \mathrm{s}, \mathrm{OCH}_{3}\right), 3.82\left(6 \mathrm{H}, \mathrm{s}, \mathrm{OCH}_{3} \times 2\right), 5.60(1 \mathrm{H}, \mathrm{s}, \mathrm{OH}), 6.90- \\
6.57(6 \mathrm{H}, \mathrm{m} \text {, aromatic } \mathrm{H}) .\end{array}$ \\
\hline $\mathrm{X}$ & $2.77\left(4 \mathrm{H}, \mathrm{s}, \mathrm{CH}_{2}-\mathrm{CH}_{2}\right), 3.84\left(3 \mathrm{H}, \mathrm{s}, \mathrm{OC} \underline{\mathrm{H}}_{3}\right), 5.56(1 \mathrm{H}, \mathrm{s}, \mathrm{O} \underline{\mathrm{H}}), 5.92\left(2 \mathrm{H}, \mathrm{s}, \stackrel{\mathrm{O}}{\mathrm{O}}>\mathrm{CH}_{2}\right) 6.84-6.56$ \\
\hline $\mathrm{XII} b)$ & $\begin{array}{l}(6 \mathrm{H}, \mathrm{m} \text {, aromatic } \mathrm{H}) . \\
2.89-2.64\left(4 \mathrm{H}, \mathrm{m}, \mathrm{CH}_{2}-\mathrm{CH}_{2}\right), 3.68\left(3 \mathrm{H}, \mathrm{s}, \mathrm{OCH}_{3}\right), 6.78-6.56(3 \mathrm{H}, \mathrm{m}, \text { aromatic } \mathrm{H}), 7.45- \\
7.34(2 \mathrm{H}, \mathrm{m}, 5 \text { and } 6 \mathrm{H}), 7.83-7.72(2 \mathrm{H}, \mathrm{m}, 2 \text { and } 4 \mathrm{H}) .\end{array}$ \\
\hline $\mathrm{XII} b)$ & $\begin{array}{l}2.92-2.65\left(4 \mathrm{H}, \mathrm{m}, \mathrm{CH}_{2}-\mathrm{CH}_{2}\right), 3.71\left(3 \mathrm{H}, \mathrm{s}, \mathrm{OCH}_{3}\right), 6.80-6.57(3 \mathrm{H}, \mathrm{m} \text {, aromatic } \mathrm{H}), 7.33(2 \mathrm{H}, \\
\mathrm{d}, J=8 \mathrm{~Hz}, 2 \text { and } 6 \mathrm{H}), 7.85(2 \mathrm{H}, \mathrm{d}, J=8 \mathrm{~Hz}, 3 \text { and } 5 \mathrm{H}) .\end{array}$ \\
\hline $\mathrm{XV}$ & $\begin{array}{l}1.36\left(3 \mathrm{H}, \mathrm{t}, J=7 \mathrm{~Hz}, \mathrm{CH}_{2}-\mathrm{CH}_{3}\right), 2.83\left(4 \mathrm{H}, \mathrm{m}, \mathrm{CH}_{2}-\mathrm{CH}_{2}\right), 3.80\left(3 \mathrm{H}, \mathrm{s}, \mathrm{OCH}_{3}\right), 4.28(2 \mathrm{H}, \mathrm{q}, J= \\
\left.7 \mathrm{~Hz}, \mathrm{CH}_{2}-\mathrm{CH}_{3}\right), 5.36(1 \mathrm{H}, \mathrm{s}, \mathrm{OH}), 6.70-6.46(3 \mathrm{H}, \mathrm{m} \text {, aromatic } \mathrm{H}), 7.33-7.22(2 \mathrm{H}, \mathrm{m}, 5 \text { and } 6 \\
\mathrm{H}), 7.86-7.72(2 \mathrm{H}, \mathrm{m}, 2 \text { and } 4 \mathrm{H}) .\end{array}$ \\
\hline XVI & $\begin{array}{l}1.35\left(3 \mathrm{H}, \mathrm{t}, J=7 \mathrm{~Hz}, \mathrm{CH}_{2}-\mathrm{CH}_{3}\right), 2.85\left(4 \mathrm{H}, \mathrm{m}, \mathrm{CH}_{2}-\mathrm{CH}_{2}\right), 3.83\left(3 \mathrm{H}, \mathrm{s}, \mathrm{OCH}_{3}\right), 4.32(2 \mathrm{H}, \mathrm{q}, \\
\left.J=7 \mathrm{~Hz}, \mathrm{CH}_{2}-\mathrm{CH}_{3}\right), 5.55(1 \mathrm{H}, \mathrm{s}, \mathrm{OH}), 6.78-6.52(3 \mathrm{H}, \mathrm{m} \text {, aromatic } \mathrm{H}), 7.18(2 \mathrm{H}, \mathrm{d}, J=8 \mathrm{~Hz}, \\
2 \text { and } 6 \mathrm{H}), 7.93(2 \mathrm{H}, \mathrm{d}, J=8 \mathrm{~Hz}, 3 \text { and } 5 \mathrm{H}) .\end{array}$ \\
\hline XVII & 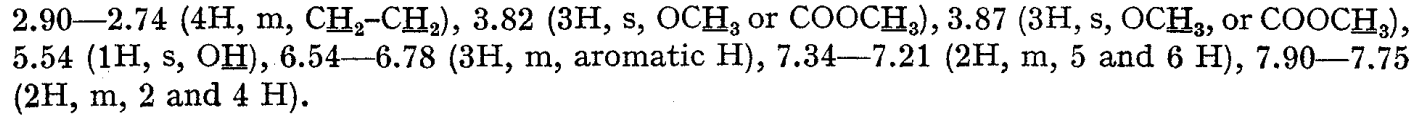 \\
\hline XVIII & $\begin{array}{l}2.93-2.77\left(4 \mathrm{H}, \mathrm{m}, \mathrm{CH}_{2}-\mathrm{CH}_{2}\right), 3.84\left(3 \mathrm{H}, \mathrm{s}, \mathrm{OCH}_{3} \text { or } \mathrm{COOCH}_{3}\right), 3.88\left(3 \mathrm{H}, \mathrm{s}, \mathrm{OCH}_{3} \text { or } \mathrm{COOCH}_{3}\right) \text {, } \\
5.56(1 \mathrm{H}, \mathrm{s}, \mathrm{OH}), 6.82-6.56(3 \mathrm{H}, \mathrm{m} \text {, aromatic } \mathrm{H}), 7.23(2 \mathrm{H}, \mathrm{d}, J=8 \mathrm{~Hz}, 2 \text { and } 6 \mathrm{H}), 7.94(2 \mathrm{H} \text {, } \\
\text { d, } J=8 \mathrm{~Hz}, 3 \text { and } 5 \mathrm{H}) \text {. }\end{array}$ \\
\hline $\mathrm{XX}$ & $\begin{array}{l}1.90(1 \mathrm{H}, \text { broad, } \mathrm{OH}), 2.83\left(4 \mathrm{H}, \mathrm{s} \text {, with shoulder, } \mathrm{CH}_{2}-\mathrm{CH}_{2}\right), 3.82\left(3 \mathrm{H}, \mathrm{s}, \mathrm{OCH}_{3}\right), 4.64(2 \mathrm{H}, \mathrm{s} \text {, } \\
\left.\mathrm{CH}_{2} \mathrm{OH}\right), 5.50(1 \mathrm{H} \text {, broad, } \underline{\mathrm{OH}}), 7.30-6.57(7 \mathrm{H}, \mathrm{m} \text {, aromatic } \mathrm{H}) .\end{array}$ \\
\hline $\mathrm{XXI} b)$ & $\begin{array}{l}2.74\left(4 \mathrm{H}, \mathrm{s} \text {, with shoulder, } \mathrm{CH}_{2}-\mathrm{CH}_{2}\right), 3.29(2 \mathrm{H}, \mathrm{s}, \mathrm{OH} \times 2), 3.70\left(3 \mathrm{H}, \mathrm{s}, \mathrm{OC} \underline{\mathrm{H}}_{3}\right), 4.43(2 \mathrm{H} ., \mathrm{s} \text {, } \\
\left.\mathrm{CH}_{2} \mathrm{OH}\right), 7.28-6.54(7 \mathrm{H}, \mathrm{m} \text {, aromatic } \mathrm{H}) .\end{array}$ \\
\hline XXII & $\begin{array}{l}2.27\left(3 \mathrm{H}, \mathrm{s}, \mathrm{CH}_{3}\right), 2.79\left(4 \mathrm{H}, \mathrm{s} \text {, with shoulder, } \mathrm{CH}_{2}-\mathrm{CH}_{2}\right), 3.82\left(3 \mathrm{H}, \mathrm{s}, \mathrm{OCH}_{3}\right), 5.45(1 \mathrm{H} \text {, broad, } \\
\mathrm{OH}), 7.13-6.58(7 \mathrm{H}, \mathrm{m} \text {, aromatic } \mathrm{H}) .\end{array}$ \\
\hline XXII & $\begin{array}{l}2.30\left(3 \mathrm{H}, \mathrm{s}, \mathrm{CH}_{3}\right), 2.82\left(4 \mathrm{H}, \mathrm{s}, \mathrm{CH}_{2}-\mathrm{CH}_{2}\right), 3.84\left(3 \mathrm{H}, \mathrm{s}, \mathrm{OC} \underline{H}_{3}\right), 5.57(1 \mathrm{H}, \mathrm{s}, \mathrm{OH}), 7.25(7 \mathrm{H}, \mathrm{m}, \\
\text { aromatic } \mathrm{H}) \text {. }\end{array}$ \\
\hline XXIV & $\begin{array}{l}2.28\left(3 \mathrm{H}, \mathrm{s}, \mathrm{CH}_{3}\right), 2.78\left(4 \mathrm{H}, \mathrm{s}, \mathrm{CH}_{2}-\mathrm{CH}_{2}\right), 3.82\left(3 \mathrm{H}, \mathrm{s}, \mathrm{OCH}_{3}\right), 5.54(1 \mathrm{H}, \mathrm{s}, \mathrm{OH}), 7.09-6.57 \\
(7 \mathrm{H}, \mathrm{m} \text {, aromatic } \mathrm{H}) \text {. }\end{array}$ \\
\hline $\mathrm{XXV}$ & $1.20\left(6 \mathrm{H}, \mathrm{d}, J=9 \mathrm{~Hz}, \mathrm{CH}\left\langle{\underset{\mathrm{CH}}{3}}_{3}^{\mathrm{CH}_{3}}\right), 2.55-3.00\left(4 \mathrm{H}, \mathrm{m}, \mathrm{CH}_{2}-\mathrm{CH}_{2}\right), 2.90-3.34\left(1 \mathrm{H}, \mathrm{m}, \mathrm{CH}\left\langle\mathrm{CH}_{3} \mathrm{CH}_{3}\right)\right.\right.$, \\
\hline XXVI & $\begin{array}{l}3.75\left(3 \mathrm{H}, \mathrm{s}, \mathrm{OCH}_{3}\right), 5.74(1 \mathrm{H}, \mathrm{s}, \mathrm{OH}), 6.55-7.38(7 \mathrm{H}, \mathrm{m} \text {, aromatic } \mathrm{H}) . \\
2.78\left(4 \mathrm{H}, \mathrm{s}, \mathrm{CH}_{2}-\mathrm{CH}_{2}\right), 3.81\left(3 \mathrm{H}, \mathrm{s}, \mathrm{OCH}_{3}\right), 5.49(1 \mathrm{H}, \text { broad, } \mathrm{OH}), 7.30-6.50(7 \mathrm{H}, \mathrm{m} \text {, aromatic } \\
\mathrm{H}) .\end{array}$ \\
\hline $\begin{array}{l}\text { XXVII } \\
\text { XXVIII }\end{array}$ & $2.77\left(4 \mathrm{H}, \mathrm{s}, \mathrm{CH}_{2}-\mathrm{CH}_{2}\right), 3.80\left(3 \mathrm{H}, \mathrm{s}, \mathrm{OCH}_{3}\right), 5.68(1 \mathrm{H}, \mathrm{s}, \mathrm{OH}), 7.19-6.52(7 \mathrm{H}, \mathrm{m}$, aromatic $\mathrm{H})$. \\
\hline$X$ & $5.58(1 \mathrm{H}, \mathrm{s}, \mathrm{OH}), 7.40-6.61(7 \mathrm{H}, \mathrm{m}$, aromatic $\mathrm{H})$ \\
\hline
\end{tabular}

a) The data were obtained on a Hitachi Model R-22 spectrometer at $90 \mathrm{MHz}$ employing tetramethylsilane as an internal standard.

b) $d_{6}$-DMSO was used as a solvent. 
2-Isopropyl- $\beta$-(3-hydroxy-4-methoxyphenyl)ethylbenzene (XXV)-2-Methoxycarbonyl-(3'-benzyloxy$4^{\prime}$-methoxy)stilbene was obtained by the reaction with 2 -carboxy-( $3^{\prime}$-benzyloxy-4'-methoxy)stilbene $(7.9 \mathrm{~g})$ and excess of diazomethane in dry benzene. The product was recrystallized from ligroin. Coloriess needles, $\mathrm{mp} 103^{\circ}, 7.5 \mathrm{~g}(92.6 \%)$. A solution of this product $(7.5 \mathrm{~g})$ in dry ether was added to an ether solution of methyl magnesium iodide prepared from methyl iodide $(28.4 \mathrm{~g})$ and magnesium $(4.8 \mathrm{~g})$, and refluxed for $2 \mathrm{hr}$. The reaction mixture was worked up in usual way to give an oily product of 2-(1-hydroxy-1-methylethyl)-3'benzyloxy-4'-methoxystilbene $(7.2 \mathrm{~g}, 95 \%)$. The oil was dissolved in ethanol and was catalytically reduced using $10 \% \mathrm{Pd} / \mathrm{C}$. The residue was purified by column chromatography using silica gel and chloroform, and was finally distilled in vacuo. Colorless oil, bp $180-182^{\circ}(4 \mathrm{mmHg}), 4.8 \mathrm{~g}(92.3 \%)$. VI.

The elemental analysis and NMR spectra of newly synthesized materials were given in Table V and Table 